\title{
Malignant salivary gland tumours: Can fast neutron therapy results point the way to carbon ion therapy?
}

Clare Stannard, Frederik Vernimmen, Henri Carrara, Dan Jones, Shaheeda Fredericks, Jos Hille and Evan de Kock

\begin{abstract}
:
Background and purpose: To evaluate the outcome of malignant salivary gland tumours treated with neutron therapy to assess the potential for other high linear energy transfer (LET) beams.
\end{abstract}

Materials and methods: Neutrons at iThemba LABS are produced by the reaction of $66 \mathrm{MeV}$ protons on a beryllium target. A median dose $20.4 \mathrm{~Gy}$, in 12 fractions in 4 weeks or 15 fractions in 5 weeks, was given to 335 patients with 176 irresectable, 104 macroscopically residual and 55 unresected tumours.

Results: Locoregional control was $60.6 \%$ at 5 years and $39.1 \%$ at 10 years and DSS was $66.8 \%$ and $53.7 \%$ at 5 and 10 years respectively.

In the univariate analysis $\mathrm{T} 4,>4 \mathrm{~cm}$, high grade, squamous carcinoma, unresected and irresectable tumours, and positive nodes were significantly worse for LRC. In the multivariate analysis tumours $>6 \mathrm{~cm}$, squamous carcinoma, irresectable tumours and nodes were significantly worse for LRC. Tumours $>6 \mathrm{~cm}$, high grade, squamous carcinoma and nodes were significantly worse for DSS. Neither LRC nor DSS was influenced by age, sex, site, dose, fractionation or for initial or recurrent disease.

Conclusions: Neutron therapy appears to be the treatment of choice for macroscopically incompletely excised and irresectable salivary gland tumours with improved survival rates. Further improvement may be achieved with other high LET modalities with a superior dose profile, such as carbon ions.

The increasing number of hadron therapy centres around the world and their use of carbon ion beams have resulted in a growing interest in high-linear energy transfer (LET) radiotherapy. Carbon ions combine the advantages of high-LET radiation with the superior ballistic properties of charged particles. The most commonly used high-LET therapy to date is neutron therapy. The rationale for using it for salivary gland tumours is based on Battermann's observations of growth delay of pulmonary metastases treated with neutrons relative to cobalt60 radiation [1]. He derived a relative biological effect (RBE) of 8 for fractionated therapy to adenoid cystic carcinoma metastases compared with 3 for most normal tissues, implying a significant therapeutic gain for salivary gland tumours. The safe application of high-LET hadron therapy requires biologically driven treatment planning that has to be based on clinical data. We contribute to this 
pool of data by reporting the results of salivary gland tumours treated with neutron therapy at iThemba LABS (formerly National Accelerator Centre), South Africa, over the last 20 years.

\section{Materials and methods}

iThemba LABS is the only heavy particle therapy facility in the Southern Hemisphere. The main accelerator is a $200 \mathrm{MeV}$ separated-sector cyclotron. It is the only facility in the world where both high-energy neutrons and highenergy protons are used for patient treatment and also provides facilities for basic and applied research and the production of medical radionuclides. Neutron therapy is available on Tuesdays, Wednesdays and Thursdays each week. Most patients are referred through one of the two local university teaching hospitals; Groote Schuur Hospital (GSH)/University of Cape Town and Tygerberg Hospital (TBH)/University of Stellenbosch.

\section{Technical and physical aspects}

The neutron therapy facility incorporates an isocentric gantry - a moving floor permits full $\mathrm{rv} 185^{\circ}$ rotation [2]. Variable rectangular field sizes between $5.5 \times 5.5$ $\mathrm{cm}^{2}$ and $29 \times 29 \mathrm{~cm}^{2}$ at a source-axis distance of $150 \mathrm{~cm}$. are available. Neutrons are produced by the reaction of $66 \mathrm{MeV}$ protons on a $1.96 \mathrm{~cm}$ thick beryllium target. Downstream of the target are several beam modification devices: steel flattening filters, tungsten wedge filters and a polyethylene hardening filter. This results in similar depth dose characteristics to an $8 \mathrm{MV}$ X-ray beam. A novel $12-\mathrm{cm}$ thick steel post-collimator multiblade trimmer is used for conformal field shaping [3]. The absorbed doses reported here are the total absorbed doses, neutron + photon. The photon component comprises less than $6 \%$ and can be neglected as the neutron RBE is about 3 .

\section{Clinical aspects}

Patients eligible for neutron therapy are those with irresectable tumours, macroscopic residual disease after surgery and resectable tumours where the functional status associated with resection was considered to be suboptimal. From February 1989 until December 2008, 401 patients were treated with curative intent, and they have been followed up until 7 February, 2012. This retrospective analysis is confined to 335 patients with malignant tumours that received a full therapeutic dose to the whole of the target volume with neutron therapy only. There were 174 males and 161 females, with an age range from 8 to 96 years, median 58 years. Tumour characteristics are shown in Table 1. Squamous cell carcinomas of the parotid and submandibular glands were included if this appeared to be the primary site. The histology was reviewed by $\mathrm{JH}$. Tumours of major salivary glands were classified according to the 2002 UICC/AJCC system. Tumours of minor salivary glands were classified according to the respective sites [4].

\section{Treatment details}

Patients were CT scanned in an immobilising head cast with $5 \mathrm{~mm}$ thick slices at increments of $5 \mathrm{~mm}$ for $3 \mathrm{D}$ planning. Prior to 2000, treatment planning was done using a Theraplan planning system at GSH and a General Electric (GE) Target Planning 
system at TBH. After 2000 an in-house developed treatment planning system, using a pencil beam model, was used [5,6]. This system uses VIRTUOS, developed by Deutches KrebsForschungZentrum (DKFZ), as a front end for the bulk of the treatment planning tasks [7]. Treatment was delivered with source-skin distance (SSD) set-ups until 2000 and thereafter isocentric set-ups were used. Prior to the introduction of the multiblade trimmer in 2000, 12-cm thick tungsten blocks provided the final beam shaping.

All neutron doses were prescribed to a minimum tumour dose encompassing the planning target volume (PTV). Up until 2002 the majority of patients received a median total absorbed dose of 20.4 Gy in 12 fractions, over 4 weeks. For those patients who had a delay in completing the prescribed course it was noticed that acute morbidity was reduced. Based on this clinical observation together with the results from radiobiological experiments in our neutron beam which showed that there was in fact repair [8], the fractionation schedule was adjusted to $20.4 \mathrm{~Gy}$ in 15 fractions over 5 weeks in 2002. Node positive patients had levels II, III and IV included in the PTV and these levels were treated adjunctively, with $14-15$ Gy in $3-5$ weeks, in $25 \%$ of node negative patients. The dose to the spinal cord was restricted to $12 \mathrm{~Gy}$ to the lateral cord and since 1992 the brain dose has been limited to $13 \mathrm{~Gy}$. Complications were scored using the RTOG scoring system [9].

\section{Statistical analysis}

Loco-regional control and disease-specific survival rates by key variables were explored using the Kaplan-Meier life table analysis. Kaplan-Meier graphs are shown to 10 years of follow-up (this period covers approximately 90\% of failures). The log rank test was used to evaluate statistical significance of differences between curves for the full dataset. Hazard Ratios (HR) and their 95\% confidence intervals (CI's) were estimated using Cox proportional hazards modelling to examine the magnitude of associations between variables. All HRs labelled "crude" are adjusted for age in single years and sex and are referred to as univariate analyses throughout the text. For multivariate analyses, all clinically relevant variables (see Table 2) were included in the model. The 95\%CI's for all proportions, rates and HRs are shown in parentheses. $P$ values were two-sided and considered significant if $p 6$ o.05. All analyses were performed using Stata software, version 12.1 (Stata Corp LP, 4905 Lakeway Drive, College Station, TX 77845, USA).

\section{Results}

\section{Local control and survival}

Local control (LC) is defined as no evidence of clinical or radiological progression of disease and loco-regional control (LRC) applies to both the primary and lymph nodes. Disease specific survival (DSS) is defined as death due to disease, locoregional, metastases or both. 
Table 1

Characteristics of malignant salivary gland tumours.

\begin{tabular}{|c|c|c|c|c|c|c|c|}
\hline \multicolumn{7}{|l|}{ Site } & 335 \\
\hline \multicolumn{7}{|c|}{ Parotid gland } & 160 \\
\hline \multicolumn{7}{|c|}{ Submandibular gland } & 26 \\
\hline \multicolumn{7}{|c|}{ Oral cavity and oropharynx } & 90 \\
\hline \multicolumn{6}{|c|}{ Hard palate } & 32 & \\
\hline & palate & & & & & 19 & \\
\hline \multicolumn{6}{|c|}{ Tongue } & 17 & \\
\hline \multicolumn{6}{|c|}{ Floor of mouth } & 8 & \\
\hline \multicolumn{6}{|c|}{ Buccal mucosa } & 6 & \\
\hline \multicolumn{6}{|c|}{ Tonsil } & 3 & \\
\hline \multicolumn{6}{|c|}{ Alveolus/mandible } & 4 & \\
\hline \multirow{2}{*}{\multicolumn{6}{|c|}{$\begin{array}{l}\text { Interalveolar space } \\
\text { Paranasal sinuses,nose, nasopharynx }\end{array}$}} & 1 & \\
\hline & & & & & & & 51 \\
\hline \multicolumn{6}{|c|}{ Maxillary antrum } & 35 & \\
\hline \multicolumn{6}{|c|}{ Ethmoid sinus } & 1 & \\
\hline \multicolumn{6}{|c|}{ Nasal cavity } & 9 & \\
\hline \multicolumn{6}{|c|}{ Nasopharynx } & 6 & \\
\hline \multicolumn{6}{|c|}{ Other } & & 8 \\
\hline \multicolumn{6}{|c|}{ Trachea } & 6 & \\
\hline \multicolumn{6}{|c|}{ Larynx } & 1 & \\
\hline & imal g & & & & & 1 & \\
\hline Histol & & & & & & & $\underline{335}$ \\
\hline Low & rade & & & & & & 57 \\
\hline & grade & ucoe & lermoi & carcinoma & & 25 & \\
\hline & grade & blyms & hous a & enocarcinon & & 22 & \\
\hline & ic cell & rcino & & & & 10 & \\
\hline Ade & id cys & carci & $\mathrm{ma}$ & & & & 108 \\
\hline Hig & irade & & & & & & 143 \\
\hline & nocarc & oma $\mathrm{s}$ & & & & 60 & \\
\hline & grade & nucoe & dermo & carcinoma & & 47 & \\
\hline & ifferen & ted $c$ & cinoma & & & 13 & \\
\hline & epithe & 1 car & oma & & & 14 & \\
\hline & inoma & x plec & orphic & denoma & & 9 & \\
\hline Squ & ous $\mathrm{c}$ & carci & & & & & 21 \\
\hline Oth & & & & & & & 6 \\
\hline & l cell & enoca & inoma & & & 1 & \\
\hline & inoma & & & & & 2 & \\
\hline & ocytic & rcino & & & & 2 & \\
\hline & tal car & oma & & & & 1 & \\
\hline Diseas & status & & & & & & \\
\hline & al dise & & & & & & 279 \\
\hline & Irrent & sease & & & & & 56 \\
\hline Surgic & status & & & & & & \\
\hline & sectabl & & & & & & 176 \\
\hline & roscop & resid & & & & & 104 \\
\hline & esecte & & & & & & 55 \\
\hline Classi & ation & & & Size & All & Macr & idual \\
\hline $\mathrm{T} 1$ & 4 & No & 247 & $0-2 \mathrm{~cm}$ & 48 & 40 & \\
\hline $\mathrm{T} 2$ & 37 & N1 & 42 & $<2-4 \mathrm{~cm}$ & 80 & 37 & \\
\hline T3 & 83 & N2 & 41 & $<4-6 \mathrm{~cm}$ & 88 & 14 & \\
\hline $\mathrm{T} 4$ & 211 & N3 & 5 & $>6 \mathrm{~cm}$ & 119 & 13 & \\
\hline Total & $\underline{335}$ & & $\underline{335}$ & & $\underline{335}$ & $\underline{104}$ & \\
\hline
\end{tabular}


Table 2

The association between key variables and loco-regional control and disease specific survival estimated by Cox's proportional hazards ratios (crude and adjusted) and their $95 \%$ confidence intervals ( $\mathrm{Cl}$ ) among 335 malignant salivary gland tumours treated with neutron therapy.

\begin{tabular}{|c|c|c|c|c|c|c|c|c|c|c|c|}
\hline \multirow[t]{2}{*}{ Variable } & \multirow[t]{2}{*}{$N$} & \multicolumn{5}{|c|}{ Loco-regional control } & \multicolumn{5}{|c|}{ Disease-specific survival } \\
\hline & & $\begin{array}{l}\text { Person } \\
\text { time } \\
\text { (months) }\end{array}$ & Failures & $\begin{array}{l}\text { Rate }(95 \% \mathrm{CI}) / \\
1000 \text { person- } \\
\text { months }\end{array}$ & Crude HR' & $\begin{array}{l}\text { Adjusted } \\
\text { HR }\end{array}$ & $\begin{array}{l}\text { Person } \\
\text { time } \\
\text { (months) }\end{array}$ & Failures & $\begin{array}{l}\text { Rate }(95 \% \mathrm{CI}) / \\
1000 \text { person- } \\
\text { months }\end{array}$ & Crude HR' & $\begin{array}{l}\text { Adjusted } \\
\text { HR }\end{array}$ \\
\hline \multicolumn{12}{|l|}{ Age group (years) } \\
\hline$<60$ & 182 & 8023.3 & 61 & $7.6(5.9-9.8)$ & $\begin{array}{l}1.0 \\
\text { (reference) }\end{array}$ & $\begin{array}{l}1.0 \\
\text { (reference) }\end{array}$ & 8987.6 & 44 & $4.9(3.6-6.6)$ & $\begin{array}{l}1.0 \\
\text { (reference) }\end{array}$ & $\begin{array}{l}1.0 \\
\text { (reference) }\end{array}$ \\
\hline $60+$ & 153 & 4396.2 & 46 & 10. $5(7.8-14.0)$ & $\begin{array}{l}1.2(0.8- \\
1.8)\end{array}$ & $\begin{array}{l}1.2(0.8- \\
1.8)\end{array}$ & 4822.9 & 41 & $8.5(6.3-11.5)$ & $\begin{array}{l}1.5(0.99- \\
2.4)\end{array}$ & $\begin{array}{l}1.5(0.9- \\
2.4)\end{array}$ \\
\hline \multicolumn{12}{|l|}{ Sex } \\
\hline Female & 161 & 6810.7 & 55 & $8.1(6.2-10.5)$ & $\begin{array}{l}1.0 \\
\text { (reference) }\end{array}$ & $\begin{array}{l}1.0 \\
\text { (reference) }\end{array}$ & 7800.9 & 38 & $4.9(3.5-6.7)$ & $\begin{array}{l}1.0 \\
\text { (reference) }\end{array}$ & $\begin{array}{l}1.0 \\
\text { (reference) }\end{array}$ \\
\hline Male & 174 & 5608.7 & 52 & $9.3(7.1-12.2)$ & $\begin{array}{l}1.1(0.8- \\
1.6)\end{array}$ & $\begin{array}{l}0.9(0.6- \\
1.4)\end{array}$ & 6009.6 & 47 & $7.8(5.9-10.4)$ & $\begin{array}{l}1.5(0.97- \\
2.3)\end{array}$ & $\begin{array}{l}1.1(0.7- \\
1.8)\end{array}$ \\
\hline \multicolumn{12}{|l|}{ Classification } \\
\hline $\mathrm{T} 1$ and $\mathrm{T}^{+}$ & 41 & 2511.6 & 7 & $2.8(1.3-5.8)$ & $\begin{array}{l}1.0 \\
\text { (reference) }\end{array}$ & $\begin{array}{l}1.0 \\
\text { (reference) }\end{array}$ & 2712.6 & 8 & $2.9(1.5-5.9)$ & $\begin{array}{l}1.0 \\
\text { (reference) }\end{array}$ & $\begin{array}{l}1.0 \\
\text { (reference) }\end{array}$ \\
\hline T3 & 83 & 3891.6 & 21 & $5.4(3.5-8.3)$ & $\begin{array}{l}1.7(0.7- \\
4.0)\end{array}$ & $\begin{array}{l}1.2(0.4- \\
3.1)\end{array}$ & 4347.5 & 15 & $3.4(2.1-5.7)$ & $\begin{array}{l}1.0(0.4- \\
2.4)\end{array}$ & $\begin{array}{l}0.5(0.2- \\
1.5)\end{array}$ \\
\hline $\mathrm{T} 4$ & 211 & 6016.3 & 79 & $13.1(10.5-16.4)$ & $\begin{array}{l}3.9(1.8- \\
8.5)\end{array}$ & $\begin{array}{l}2.1(0.9- \\
5.2)\end{array}$ & 6750.5 & 62 & $9.2(7.2-11.8)$ & $\begin{array}{l}2.4(1.2- \\
5.1)\end{array}$ & $\begin{array}{l}0.8(0.3- \\
2.1)\end{array}$ \\
\hline \multicolumn{12}{|l|}{ Size $(\mathrm{cm})$} \\
\hline $0-2$ & 48 & 3134.0 & 9 & $2.9(1.5-5.5)$ & $\begin{array}{l}1.0 \\
\text { (reference) }\end{array}$ & $\begin{array}{l}1.0 \\
\text { (reference) }\end{array}$ & 6376.1 & 9 & $2.7(1.4-5.3)$ & $\begin{array}{l}1.0 \\
\text { (reference) }\end{array}$ & $\begin{array}{l}1.0 \\
\text { (reference) }\end{array}$ \\
\hline $2-4$ & 80 & 4242.5 & 22 & $5.2(3.4-7.9)$ & $\begin{array}{l}1.8(0.8- \\
3.8)\end{array}$ & $\begin{array}{l}2.0(0.8- \\
4.7)\end{array}$ & 4733.3 & 13 & $2.7(1.6-4.7)$ & $\begin{array}{l}1.0(0.4- \\
2.4)\end{array}$ & $\begin{array}{l}1.2(0.4- \\
3.0)\end{array}$ \\
\hline 4-6 & 88 & 2793.6 & 38 & $13.6(9.9-18.7)$ & $\begin{array}{l}4.0(1.9- \\
8.4)\end{array}$ & $\begin{array}{l}2.1(0.9- \\
5.2)\end{array}$ & 3363.7 & 28 & $8.3(5.7-12.1)$ & $\begin{array}{l}2.5(1.2- \\
5.4)\end{array}$ & $\begin{array}{l}1.8(0.7- \\
4.7)\end{array}$ \\
\hline$>6$ & 119 & 2249.4 & 38 & $16.9(12.3-23.2)$ & $\begin{array}{l}5.1(2.4- \\
10.9)\end{array}$ & $\begin{array}{l}2.6(1.01- \\
6.5)\end{array}$ & 2437.4 & 35 & $14.4(10.3-20.0)$ & $\begin{array}{l}3.7(1.8- \\
8.0)\end{array}$ & $\begin{array}{l}2.8(1.03- \\
7.6)\end{array}$ \\
\hline \multicolumn{12}{|l|}{ Site } \\
\hline $\begin{array}{l}\text { Oral cavity/ } \\
\text { oropharynx }\end{array}$ & 90 & 4374.1 & 32 & $7.3(5.2-10.3)$ & $\begin{array}{l}1.0 \\
\text { (reference) }\end{array}$ & $\begin{array}{l}1.0 \\
\text { (reference) }\end{array}$ & 5125.5 & 22 & $4.3(2.8-6.5)$ & $\begin{array}{l}1.0 \\
\text { (reference) }\end{array}$ & $\begin{array}{l}1.0 \\
\text { (reference) }\end{array}$ \\
\hline Parotid gland & 160 & 5283.9 & 42 & $7.9(5.9-10.8)$ & $\begin{array}{l}1.0(0.6- \\
1.6)\end{array}$ & $\begin{array}{l}0.9(0.5- \\
1.7)\end{array}$ & 5692.2 & 35 & $6.1(4.4-8.6)$ & $\begin{array}{l}1.1(0.6- \\
1.9)\end{array}$ & $\begin{array}{l}0.7(0.4- \\
1.5)\end{array}$ \\
\hline $\begin{array}{l}\text { Sinuses/nose/ } \\
\text { PNS }\end{array}$ & 51 & 1553.2 & 20 & $12.9(8.3-20.0)$ & $\begin{array}{l}1.6(0.9- \\
2.7)\end{array}$ & $\begin{array}{l}1.4(0.7- \\
2.7)\end{array}$ & 1756.3 & 16 & $9.1(5.6-14.9)$ & $\begin{array}{l}1.8(0.9- \\
3.5)\end{array}$ & $\begin{array}{l}1.4(0.7- \\
3.2)\end{array}$ \\
\hline $\begin{array}{l}\text { Submandibular } \\
\text { gland }\end{array}$ & 26 & 886.1 & 11 & $12.4(6.9-22.4)$ & $\begin{array}{l}1.4(0.7- \\
2.8)\end{array}$ & $\begin{array}{l}0.8(0.4- \\
2.0)\end{array}$ & 909.3 & 10 & $11.0(5.9-20.4)$ & $\begin{array}{l}1.9(0.9- \\
4.0)\end{array}$ & $\begin{array}{l}0.8(0.4- \\
2.1)\end{array}$ \\
\hline Other & 8 & 322.0 & 2 & $6.2(1.6-24.8)$ & - & - & 327.3 & 2 & $6.1(1.5-24.4)$ & - & - \\
\hline \multicolumn{12}{|l|}{ Histology } \\
\hline Low grade & 57 & 3084.4 & 13 & $4.2(2.4-7.3)$ & $\begin{array}{l}1.0 \\
\text { (reference) }\end{array}$ & $\begin{array}{l}1.0 \\
\text { (reference) }\end{array}$ & 3163.0 & 9 & $2.8(1.4-5.4)$ & $\begin{array}{l}1.0 \\
\text { (reference) }\end{array}$ & $\begin{array}{l}1.0 \\
\text { (reference) }\end{array}$ \\
\hline $\begin{array}{l}\text { Adenoid cystic } \\
\text { ca }\end{array}$ & 108 & 4318.1 & 37 & $8.5(6.2-11.8)$ & $\begin{array}{l}1.7(0.9- \\
10.4)\end{array}$ & $\begin{array}{l}1.9(0.9- \\
3.7)\end{array}$ & 5178.2 & 22 & $4.2(2.8-6.4)$ & $\begin{array}{l}1.4(0.6- \\
3.1)\end{array}$ & $\begin{array}{l}1.4(0.6- \\
3.2)\end{array}$ \\
\hline High grade & 143 & 4313.9 & 46 & $10.7(8.0-14.2)$ & $\begin{array}{l}2.2(1.2- \\
4.1)\end{array}$ & $\begin{array}{l}1.9(0.97- \\
3.7)\end{array}$ & 4706.2 & 44 & $9.3(7.0-12.6)$ & $\begin{array}{l}2.7(1.3- \\
5.5)\end{array}$ & $\begin{array}{l}2.2(1.01- \\
4.7)\end{array}$ \\
\hline Squamous ca & 21 & 406.4 & 10 & $24.6(13.2-45.7)$ & $\begin{array}{l}4.4(1.9- \\
10.2)\end{array}$ & $\begin{array}{l}4.5(1.7- \\
12.0)\end{array}$ & 426.0 & 10 & $23.5(12.6-43.6)$ & $\begin{array}{l}5.2(2.1- \\
13.1)\end{array}$ & $\begin{array}{l}4.2(1.4- \\
12.1)\end{array}$ \\
\hline Other & 6 & 296.6 & 1 & $3.4(0.5-23.9)$ & - & - & 337.1 & 0 & 0 & - & - \\
\hline \multicolumn{12}{|l|}{ Surgical status } \\
\hline $\begin{array}{l}\text { Macroscopic } \\
\text { residual }\end{array}$ & 104 & 5270.9 & 17 & $3.2(2.0-5.2)$ & $\begin{array}{l}1.0 \\
\text { (reference) }\end{array}$ & $\begin{array}{l}1.0 \\
\text { (reference) }\end{array}$ & 5409.4 & 14 & $2.6(1.5-4.4)$ & $\begin{array}{l}1.0 \\
\text { (reference) }\end{array}$ & $\begin{array}{l}1.0 \\
\text { (reference) }\end{array}$ \\
\hline Unresected & 55 & 3047.2 & 20 & $6.6(4.2-10.2)$ & $\begin{array}{l}2.0(1.04- \\
3.9)\end{array}$ & $\begin{array}{l}1.7(0.8- \\
3.7)\end{array}$ & 3657.8 & 12 & $3.3(1.9-5.8)$ & $\begin{array}{l}1.2(0.5- \\
2.6)\end{array}$ & $\begin{array}{l}0.9(0.4- \\
2.1)\end{array}$ \\
\hline Irresectable & 176 & 4101.4 & 70 & $17.1(13.5-21.6)$ & $\begin{array}{l}4.7(2.7- \\
8.0)\end{array}$ & $\begin{array}{l}2.3(1.1- \\
4.6)\end{array}$ & 4743.3 & 59 & $12.4(9.6-16.0)$ & $\begin{array}{l}3.8(2.1- \\
6.9)\end{array}$ & $\begin{array}{l}1.6(0.7- \\
3.6)\end{array}$ \\
\hline \multicolumn{12}{|l|}{ Status } \\
\hline Initial & 279 & 10386.7 & 88 & $8.5(6.9-10.4)$ & $\begin{array}{l}1.0 \\
\text { (reference) }\end{array}$ & $\begin{array}{l}1.0 \\
\text { (reference) }\end{array}$ & 11633.5 & 74 & $6.3(5.1-8.0)$ & $\begin{array}{l}1.0 \\
\text { (reference) }\end{array}$ & $\begin{array}{l}1.0 \\
\text { (reference) }\end{array}$ \\
\hline Recurrent & 56 & 2032.8 & 19 & $9.3(6.0-14.6)$ & $\begin{array}{l}1.3(0.8- \\
2.2)\end{array}$ & $\begin{array}{l}1.4(0.8- \\
2.6)\end{array}$ & 2177.0 & 11 & $5.0(2.8-9.1)$ & $\begin{array}{l}0.9(0.5- \\
1.8)\end{array}$ & $\begin{array}{l}1.1(0.5- \\
2.2)\end{array}$ \\
\hline \multicolumn{12}{|l|}{ Nodes } \\
\hline Negative & 247 & 10662.8 & 71 & $6.6(5.3-8.4)$ & $\begin{array}{l}1.0 \\
\text { (reference) }\end{array}$ & $\begin{array}{l}1.0 \\
\text { (reference) }\end{array}$ & 11873.8 & 50 & $4.2(3.2-5.5)$ & $\begin{array}{l}1.0 \\
\text { (reference) }\end{array}$ & $\begin{array}{l}1.0 \\
\text { (reference) }\end{array}$ \\
\hline Positive & 88 & 1756.6 & 36 & $20.5(14.8-28.4)$ & $\begin{array}{l}2.7(1.8- \\
4.1)\end{array}$ & $\begin{array}{l}3.0(1.8- \\
5.1)\end{array}$ & 1936.7 & 35 & $18.1(13.0-25.2)$ & $\begin{array}{l}3.2(2.0- \\
5.0)\end{array}$ & $\begin{array}{l}3.5(2.0- \\
6.1)\end{array}$ \\
\hline \multicolumn{12}{|l|}{ Dose (Gy) } \\
\hline$>20$ & 309 & 11468.9 & 95 & $8.3(6.8-10.1)$ & $\begin{array}{l}1.0 \\
\text { (reference) }\end{array}$ & $\begin{array}{l}1.0 \\
\text { (reference) }\end{array}$ & 12772.8 & 74 & $5.8(4.6-7.3)$ & $\begin{array}{l}1.0 \\
\text { (reference) }\end{array}$ & $\begin{array}{l}1.0 \\
\text { (reference) }\end{array}$ \\
\hline
\end{tabular}




\begin{tabular}{|c|c|c|c|c|c|c|c|c|c|c|c|}
\hline \multirow[t]{2}{*}{ Variable } & \multirow[t]{2}{*}{$N$} & \multicolumn{5}{|c|}{ Loco-regional control } & \multicolumn{5}{|c|}{ Disease-specific survival } \\
\hline & & $\begin{array}{l}\text { Person } \\
\text { time } \\
\text { (months) }\end{array}$ & Failures & $\begin{array}{l}\text { Rate }(95 \% \mathrm{CI}) / \\
1000 \text { person- } \\
\text { months }\end{array}$ & Crude HR ${ }^{*}$ & $\begin{array}{l}\text { Adjusted } \\
\text { HR }\end{array}$ & $\begin{array}{l}\text { Person } \\
\text { time } \\
\text { (months) }\end{array}$ & Failures & $\begin{array}{l}\text { Rate }(95 \% \mathrm{Cl}) / \\
1000 \text { person- } \\
\text { months }\end{array}$ & Crude HR ${ }^{*}$ & $\begin{array}{l}\text { Adjusted } \\
\text { HR }\end{array}$ \\
\hline $18-20$ & 26 & 950.5 & 12 & $12.6(7.2-22.2)$ & $\begin{array}{l}1.5(0.8- \\
2.7)\end{array}$ & $\begin{array}{l}1.4(0.7- \\
3.0)\end{array}$ & 1037.8 & 11 & $10.6(5.9-19.1)$ & $\begin{array}{l}1.8(0.94- \\
3.4)\end{array}$ & $\begin{array}{l}1.7(0.8- \\
3.6)\end{array}$ \\
\hline \multicolumn{12}{|l|}{ Fractions } \\
\hline 12 in 4 weeks & 201 & 8212.2 & 69 & $8.4(6.6-10.6)$ & $\begin{array}{l}1.0 \\
\text { (reference) }\end{array}$ & $\begin{array}{l}1.0 \\
\text { (reference) }\end{array}$ & 9185.4 & 55 & $5.9(4.6-7.8)$ & $\begin{array}{l}1.0 \\
\text { (reference) }\end{array}$ & $\begin{array}{l}1.0 \\
\text { (reference) }\end{array}$ \\
\hline 12 in $>4$ weeks & 44 & 1502.1 & 16 & $10.6(6.5-17.4)$ & $\begin{array}{l}1.1(0.7- \\
1.6)\end{array}$ & $\begin{array}{l}1.1(0.6- \\
2.0)\end{array}$ & 1746.8 & 12 & $6.9(3.9-12.1)$ & $\begin{array}{l}1.1(0.6- \\
2.0)\end{array}$ & $\begin{array}{l}1.1(0.5- \\
2.1)\end{array}$ \\
\hline 15 in 5 weeks & 86 & 2640.3 & 22 & $8.3(5.5-12.6)$ & $\begin{array}{l}0.8(0.5- \\
1.4)\end{array}$ & $\begin{array}{l}0.6(0.4- \\
1.1)\end{array}$ & 2813.4 & 17 & $6.0(3.8-9.7)$ & $\begin{array}{l}0.8(0.4- \\
1.3)\end{array}$ & $\begin{array}{l}0.7(0.4- \\
1.3)\end{array}$ \\
\hline Other & 4 & 64.9 & 0 & 0 & - & - & 64.9 & 1 & $15.4(2.1-109.4)$ & - & - \\
\hline
\end{tabular}

Statistically significant findings shown in bold

HR: Hazard Ratio.

- Adjusted for age (single years) and sex.

${ }^{+} \mathrm{T} 1$ and $\mathrm{T} 2$ classifications were combined since there were no failures for $\mathrm{T} 1$ (loco-regional control: $\mathrm{T} 1, n=4,257.5$ person months, rate $=0.0$, T2, $n=37,2254.1$ person months, rate $=3.1(1.5-6.5)$; disease specific survival: $\mathrm{T} 1, n=4,257.5$ person months, rate $=0.0, \mathrm{~T} 2, n=37,2455.1$ person months, rate $=3.3(1.6-6.5)$

Overall LC was $58.6 \%(51.1-65.4)$ at 5 years and $45.9 \%(36.5-54.8)$ at 10 years, and LRC was $60.6 \%(53.0-67.2)$ at 5 years and $39.1 \%(30.2-47.9)$ at 10 years, Fig. 1 . The 5and 10-year DSS was 66.8\% (59.8-72.9) and 53.7\% (44.4-62.2), and overall survival (OS) rates were $51 \%(44.3-57.3)$ and $37.4 \%(29.8-44.9)$ at 5 and 10 years respectively, Fig. 1.

Patients who died of disease, 85, had a median follow-up of 19 months (range 1-187 months); those that died of other causes, 56, had a median follow-up of 14 months (range 1-218 months); and those who were last seen alive, 194, had a median followup of 31 months (range 1-257 months).

Salvage surgery was performed on 19 patients with recurrent or progressive disease, 7 (12.7\%) unresected disease, 6 (5.8\%) macroscopic residual and 6 (3.4\%) irresectable disease.

$P$-values from log rank tests and Cox HR were calculated for each of the variables for LC and LRC, and also for DSS and OS. As there was no meaningful difference between them, univariate (Crude HR) and multivariate analyses (Adjusted HR) of LRC and DSS only are reported, Table 2.

The histological subtypes were grouped into low grade, high grade, adenoid cystic carcinoma (ACC) or squamous carcinoma. 


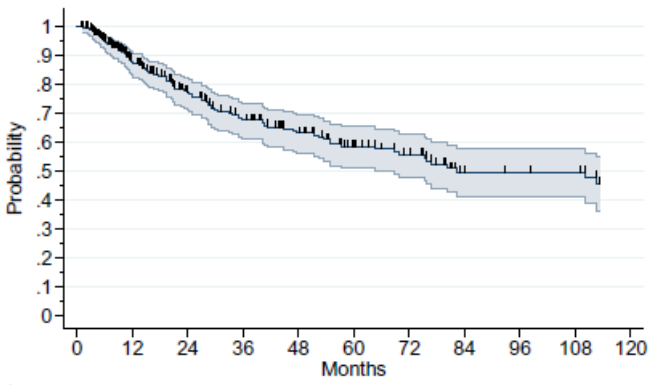

Number at risk $\begin{array}{lllllllllll}335 & 204 & 149 & 117 & 91 & 67 & 55 & 31 & 30 & 29 & 23\end{array}$

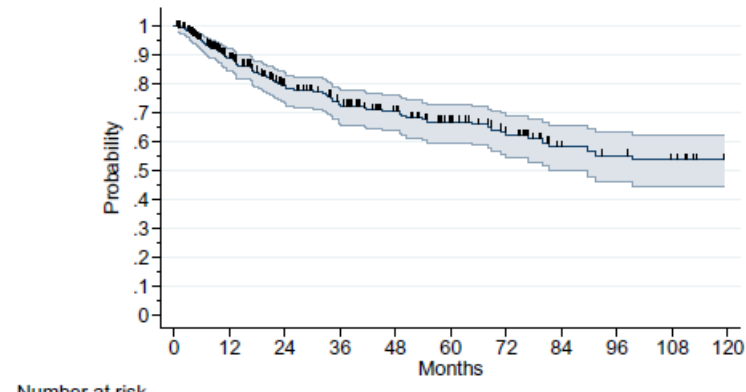

Number at risk

$\begin{array}{lllllllllll}335 & 213 & 160 & 131 & 104 & 79 & 63 & 39 & 36 & 33 & 27\end{array}$

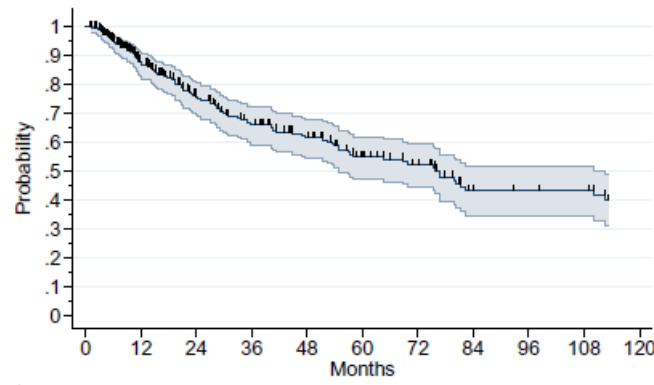

Number at risk

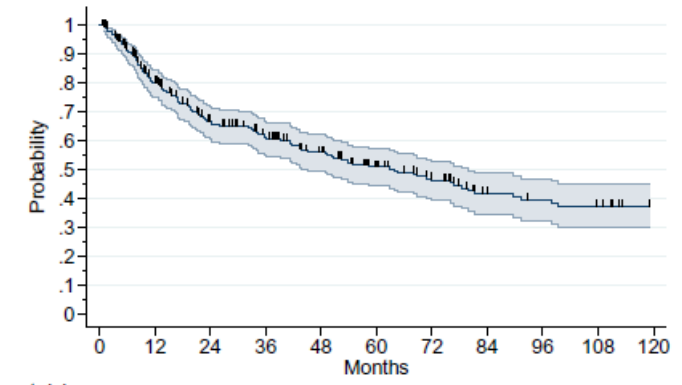

Number at risk

Fig. 1. Kaplan-Meier graphs showing local control (LC), loco-regional control (LRC), disease-specific survival (DSS) and overall survival (OS) of 335 patients with malignant salivary gland tumors treated with neutron therapy.
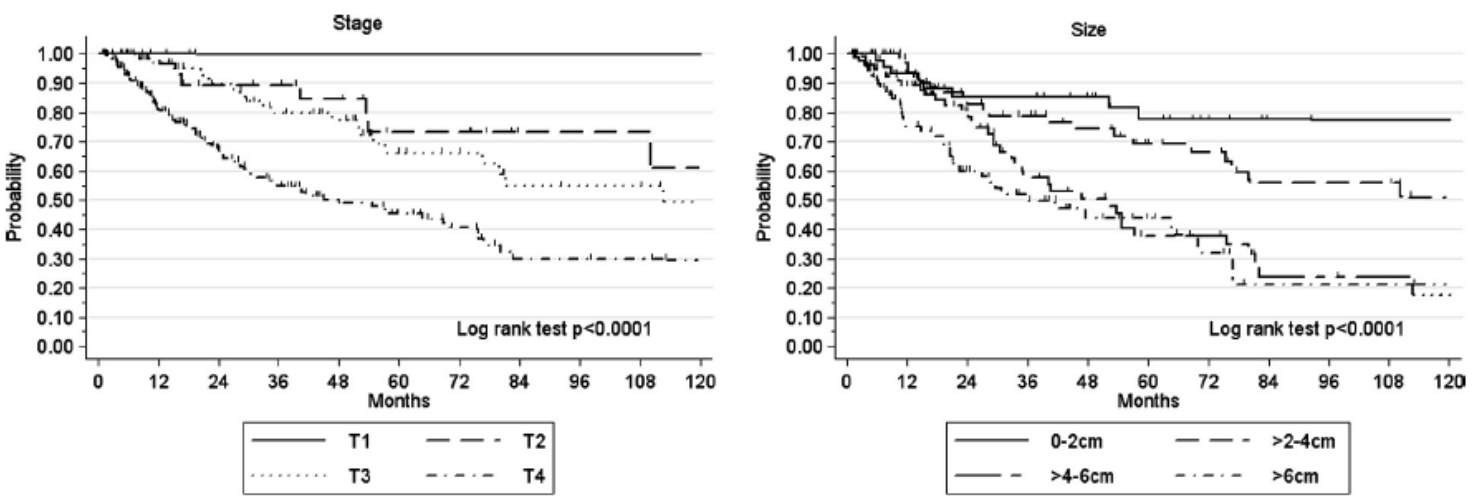

Histology
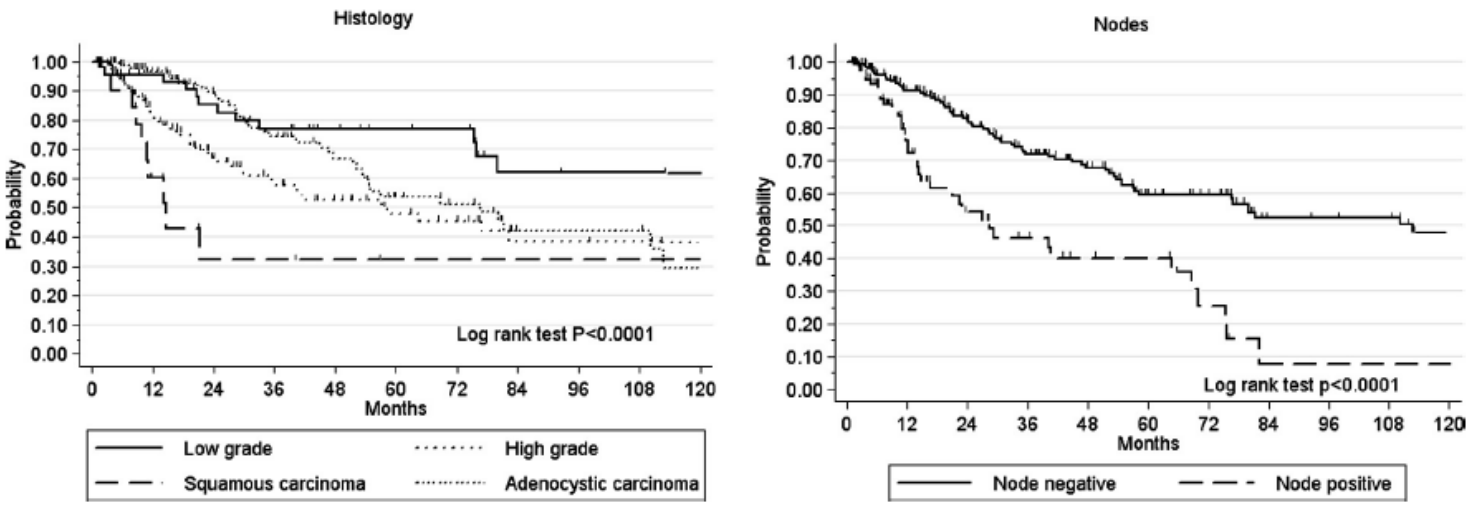

Fig. 2. Kaplan-Meier graphs showing loco-regional control (LRC) according to T classification, size, histological grade and nodal status. 
In the univariate analysis $\mathrm{T} 4$, size $>4 \mathrm{~cm}$, high grade, squamous carcinoma, unresected and irresectable tumours, and positive nodes were significantly worse for LRC, Figs. 2 and 3, and also for DSS with the exception of unresected tumours, Table 2. Salvage surgery in 7 (12.7\%) of these patients resulted in the DSS approaching those with macroscopic residual disease, Fig. 3 .

In the multivariate analysis tumours $>6 \mathrm{~cm}$, squamous carcinoma, irresectable tumours and positive nodes were significantly worse for LRC and tumours $>6 \mathrm{~cm}$, high grade, squamous carcinoma and positive nodes were significantly worse for DSS, Table 2. Neither LRC nor DSS was influenced significantly by age, sex, site, dose, fractionation or whether initial or recurrent disease was treated, Table 2.

\section{Nodal disease}

Of the 88 patients with nodes, 9 had neck dissections prior to treatment and in the other 79 patients there were 52 with a CR, 25 with stable disease and 2 with progressive disease. Fifteen of 247(6\%) No patients developed nodes; 12 outside the treated volume and 3 inside.
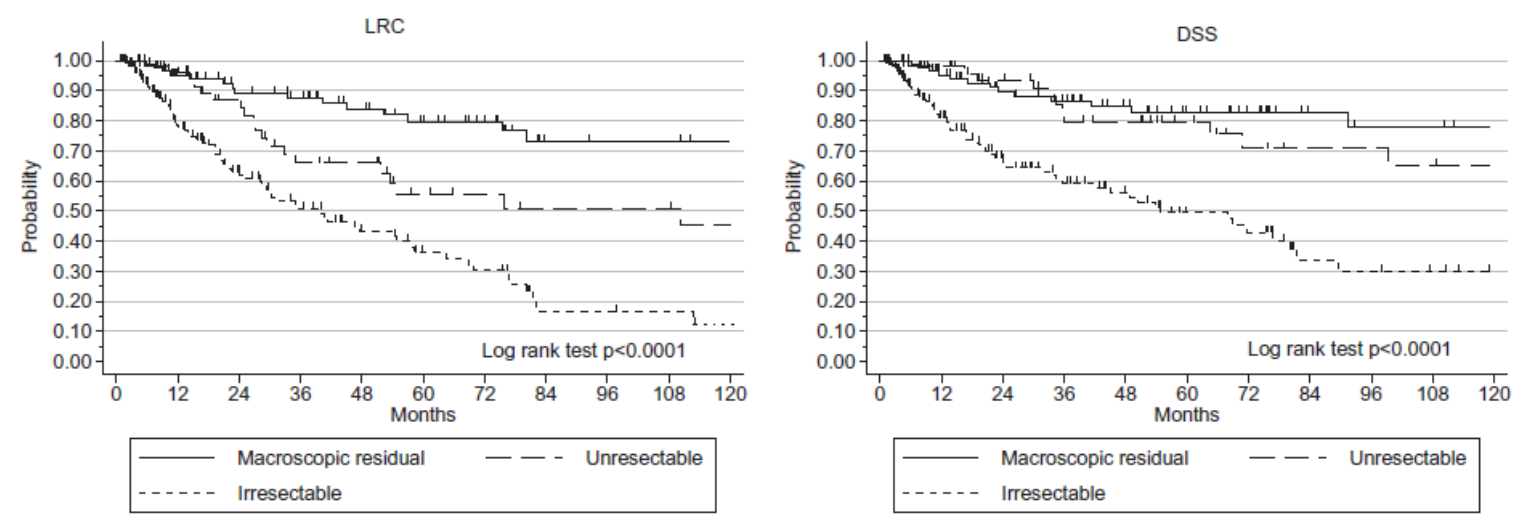

Fig. 3. Kaplan-Meier graphs showing loco-regional control (LRC) and disease specific survival (DSS) according to surgical status.

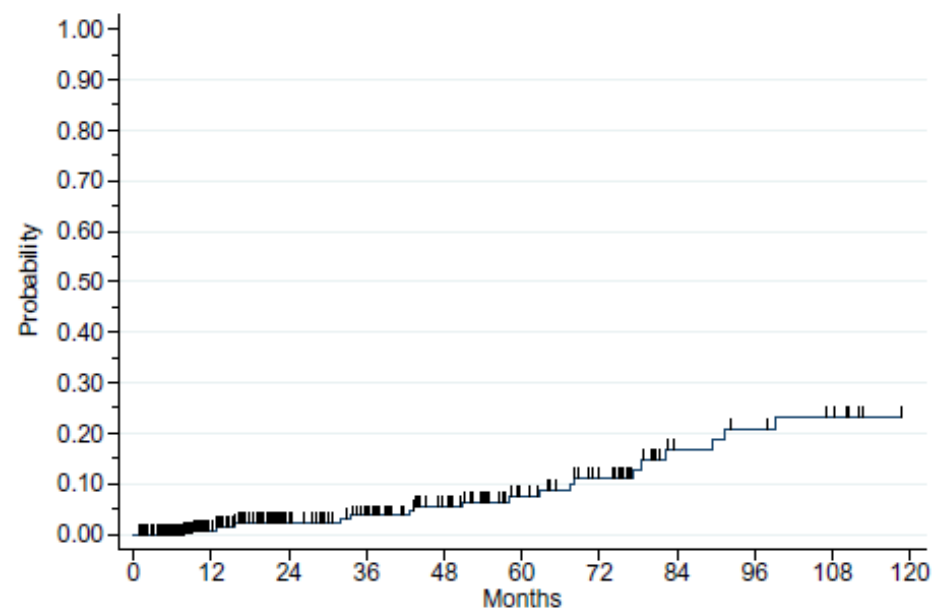

Fig. 4. Kaplan-Meier graph showing the probability of Grades 3 and 4 morbidity.

Nine died, 8 of loco-regional recurrence (LRR) and 3 with metastases in addition. Both LRC and DSS were significantly worse in node positive patients but there was no significant difference whether the nodal status was N1, N2 or N3 classification. 


\section{Distant metastases}

Metastases were present in 19 patients prior to treatment. Ten were alive when last seen, from 1 to 79 months, median 27 months, 6 died of metastases, together with LRR in 4, 1 died of LRR alone, two died of unknown causes with a clear primary site and one was lost to follow-up. Metastases developed in 59 patients; 32 died of metastases, with LR progression in 18 of them, 5 died of progressive local disease alone, one died of other causes and 21 were alive when last seen, from 11-176 months, median 44 months. Therefore only 38 of 78 patients, $49 \%$, with metastases were known to have died of them, whilst a further 6 patients died of locoregional disease. Metastases occurred in 38 patients with ACC (48\%), 20 patients with adenocarcinoma and 21 other histological subtypes. Of those patients who died 16 each (34\%) were ACC and adenocarcinoma and of those alive when last seen 22 (69\%) had ACC.

\section{Complications}

Late morbidity, greater than Grade 2, was seen in 30 patients; 13 patients Grade 3, 16 Grade 4 and 1 Grade 5. Five patients had 2 complications in which case the highest was reported. There were 3 cases of brain necrosis, 2 Grade 4 and one Grade 5 occurring in patients treated prior to 1992. One Grade 4 patient was treated successfully with surgery; the other died, either of necrosis or metastases. No incidence of CNS morbidity occurred with the brain dose limited to $13 \mathrm{~Gy}$. There was no incidence of spinal cord damage. Of the 14 other patients with Grade 4 morbidity there were 11 bone necroses, (4 of which had bone invasion initially) with or without overlying skin or mucosal ulceration, 2 skin ulcers and one mucosal ulcer. The 13 Grade 3 complications included 9 with severe xerostomia, 3 with severe subcutaneous induration and one recurrent ear infection.

The overall number of complications was 30 in $12218.5 \mathrm{pm}$ giving a complication rate of $2.4(1.7-3.5)$. Grade 3 and 4 complications were analysed in relation to dose and fractionation. There were 2 complications in $950.5 \mathrm{pm}$ in the $18-20$ Gy group and 28 in $11468.9 \mathrm{pm}$ in the $>20$ Gy group, giving complication rates of $2.1(0.5-8.5)$ and 2.5(1.7-3.6), respectively. There were 25 complications in $7749.4 \mathrm{pm}$ in the 12 fractions in 4 weeks group, 3 in $1608.3 \mathrm{pm}$ in the 12 fractions in $>4$ weeks group and 2 in $2795.9 \mathrm{pm}$ in the 15 fractions in 5 weeks group, giving complication rates of $3.2(2.2-4.8), 1.9(0.6-5.8)$ and $0.7(0.2-2.9)$ respectively. These were not statistically significant. The probability of developing complications is shown in Fig. 4.

\section{Discussion}

High-LET neutron therapy has several radiobiological advantages over low-LET radiation due to the increased energy deposition per unit length of track [10]. It has a low oxygen enhancement ratio (OER), a reduction in variation of sensitivity around the cell cycle and a decreased ability of tumour cells to repair sublethal and potentially lethal damage. Advanced salivary gland tumours are large and may contain a significant number of hypoxic cells which are relatively resistant to low-LET radiation. They also tend to be slow growing with a large proportion of cells in the resting phase of the cell cycle where they may be protected from the full effects of low-LET radiation. They 
should thus respond well to high-LET irradiation. Treatment with conventional radiation alone has not been very satisfactory, with a combined overall local control rate of $26 \%$ in 299 patients [11]. More recently, Chen showed 5-year and 10-year local control rates of $70 \%$ and $57 \%$ respectively but only $58 \%$ and $39 \%$ respectively for $\mathrm{T}_{3} / 4$ tumours [12]; Mendenhall had a 10-year local control rate of $42 \%$ but this was only $21 \%$ for T4 tumours [13]; Terhaard showed a 5-year local control rate of 50\% provided they received P66 Gy [14]. Accelerated hyperfractionated radiation was investigated by Wang and Goodman giving a median dose of $68.2 \mathrm{~Gy}$ and produced local control rates of $100 \%$ for 9 parotid tumours and $78 \%$ for 15 minor salivary gland tumours [15].

The combined overall local control rate for advanced salivary gland tumours treated with fast neutron therapy was $67 \%$ in 309 patients [11], and the randomised RTOG/MRC clinical trial confirmed the superiority of neutron therapy for local control compared to photons [16]. Subsequent results from Seattle have shown a $59 \%$ LRC rate and $67 \%$ cause specific survival (CSS) at 6 years [17] and $76.5 \%$ LC and 68.2\% CSS at 7 years in major salivary gland tumours, the majority of which had macroscopically resected disease [18]. Huber has shown a 75\% 5-year LCR rate for advanced adenoid cystic carcinomas [19].

Our results show a similar LC probability and DSS despite more irresectable disease. The survival rate is much higher than the RTOG/MRC trial. Thirty-one patients were still alive with metastatic disease when last seen. Most salivary gland tumours are slow growing as are the metastases, particularly of adenoid cystic carcinomas, and the presence of metastases when first seen should not preclude radical treatment for the primary tumour. Neutron therapy was effective in controlling nodal disease but its presence affected both LRC and survival adversely.

The incidence of severe late toxicity, Grades 3 and 4, is 30 in 335 patients (8.9\%) or 11.1\% at 6 years, Fig. 4, which is not excessive. Huber found in his comparison of neutron therapy versus mixed beam versus photon therapy that although the complication rates were higher with neutron therapy, 5 of 27 (17\%) versus 2 of 21 (10\%) versus 1 of 25(4\%) respectively, this was not significant [19]. Douglas had a 6 year actuarial Grade 3 and 4 toxicity of $10 \%$ [17]. There was no significant difference in complication rates between the different dose and fractionation groups.

Intensity modulated RT (IMRT) has the potential to treat with less morbidity but this was not borne out in the one series treating 25 patients with adenoid cystic carcinomas [20]. A median dose of $66 \mathrm{~Gy}$ was given to the CTV. Local control rate was $38 \%$ at 3 years and PFS 48\%. Neutron IMRT is theoretically possible but has not been used clinically to date. Very promising results have been achieved treating skull base adenoid cystic carcinomas with protons and photons to a median combined dose of $76 \mathrm{~Gy}$. LC was $93 \%$ at 5 years and $82 \%$ at 10 years, although the disease free survival was $56 \%$ at 5 years [21]. There were, however, 10 Grade 3 and 2 Grade 5 brain toxicities in 23 patients. Neither of these modalities has the advantages of high-LET radiation. 
Carbon ions offer the best of both worlds with their excellent dose distribution profile and similar radiobiological advantages to neutrons and early results are available from the Heavy Ion Medical Accelerator in Chiba, Japan (HIMAC) and Heidelberg, Germany. Local control of advanced head and neck tumours treated in a phase II trial with carbon ions was $73 \%$ at 5 years for both adenoid cystic carcinomas and adenocarcinomas and late morbidity did not exceed Grade 2 [22]. In another phase I/II trial locally advanced adenoid cystic carcinomas were treated with fractionated stereotactic RT or IMRT to $54 \mathrm{~Gy}$ and a carbon ion boost of 18 Gy(RBE). LRC probability was $77.5 \%$ at 4 years also with minimal morbidity [23].

As there are few centres offering neutron therapy and taking into account the rarity of salivary gland tumours, it is unlikely that any other trials will be conducted. We therefore have to rely on retrospective studies to assess the value of neutron therapy for these tumors. The results are very promising for advanced disease and neutron beam therapy should still be offered where available. Salivary gland tumours are a good indication for heavy charged particles and based on the neutron therapy response they would be an ideal pathology to investigate the value of high LET radiation. An internationally collaborative study comparing protons with carbon ions would address the question of whether high LET radiation is intrinsically better for salivary gland tumours; an important question in the light of the super high cost of carbon ion facilities [24].

\section{Funding}

There was no source of funding.

\section{Ethical considerations}

Approval for the retrospective study has been granted by

1. The Human Research Ethics Committee, Faculty of Health Sciences, University of Cape Town.

2. South African Medical Association Research Ethics Committee.

\section{Informed consent}

All cases were discussed at a multidisciplinary meeting, the consensus recommendation for neutron therapy was explained to the patient and informed consent was obtained.

\section{Conflicts of Interest Statement}

There was no conflict of interest.

\section{Acknowledgements}

The authors gratefully acknowledge Wolfgang Sauerwein, John Gueulette and Kobus Slabbert for their comments on the manuscript. 


\section{References}

[1] Battermann JJ, Breur K, Hart G. Observations on pulmonary metastases in patients after single doses and multiple fractions of fast neutrons and Cobalt-6o gamma rays. Eur J Cancer 1981;5:539-48.

[2] Jones DTL, Yudelev M, Hendrikse WLJ. Physical characteristics of the South African high energy neutron therapy facility. Radiat Prot Dosimetry 1988;23:365-8.

[3] Jones DTL, Schreuder AN, Symons JE, Binns PJ. Experimental investigations of a multiblade trimmer for neutron therapy. J Brachyther Int 1997;13:59-66.

[4] Greene FL, Page DL, Fleming ID, et al., editors. AJCC cancer staging manual. New York: Springer; 2002. pp. 23-75.

[5] de Kock EA. Pencil beam convolution model for fast-dose calculations in uncharged particle radiation treatment planning. Radiat Phys Chem 2004;71:8. [6] de Kock EA. Treatment planning system, iThemba LABS annual report; 2009, p. 63-65 (http://www.tlabs.ac.za/pdf/Annual_reports/Arepo8-o9FINAL.pdf).

[7] Bendl R, Pross J, Schlegel W. VIRTUOS-A program for VIRTUal radiOtherapy Simulation. In: Computer assisted radiology - proceedings of the international symposium CAR, vol. 93; 1993. p. 676-682.

[8] Slabbert JP, Hough JH, Jones HL, et al. Cellular damage in response to variations in the secondary charged particle spectrum of a $\mathrm{p}(66) / \mathrm{Be}$ neutron beam. NAC, annual report; 1991, NAC/AR/91-01. p. 99.

[9] http://www.rtog.org/members/toxicity/main.html.

[10] Hall EJ, editor. In radiobiology for the radiologist. Philadelphia: Lippincott Williams \& Williams; 2000. 112-123, 443.

[11] Buchholz TA, Laramore GE, Griffin BW, Koh W-J, Griffin TW. The role of fast neutron radiation therapy in the management of advanced salivary gland malignant neoplasms. Cancer 1992;69:2779-88.

[12] Chen AM, Bucci MK, Quivey JM, Garcia J, Eisele DW, Fu KK. Long-term outcome of patients treated with by radiation therapy alone for salivary gland carcinomas. Int J Radiat Oncol Biol Phys 2006;66:1044-50.

[13] Mendenhall WM, Morris CG, Amdur RJ, Werning JW, Villaret DB. Radiotherapy alone or combined with surgery for salivary gland carcinoma. Cancer 2005;103:2544-50.

[14] Terhaard $\mathrm{CH}$, Lubsen $\mathrm{H}$, Rasch $\mathrm{CR}$, et al. The role of radiotherapy in the treatment of salivary gland tumours. Int J Radiat Oncol Biol Phys 2005;61:103-11.

[15] Wang CC, Goodman M. Photon irradiation of unresectable carcinomas of salivary glands. Int J Radiat Oncol Biol Phys 1991;21:569-76.

[16] Laramore GE, Krall JM, Griffin TW, et al. Neutron vs photon irradiation for irresectable salivary gland tumors. Final report of an RTOC-MRC randomised clinical trial. Int J Radiat Oncol Biol Phys 1993;27:235-40.

[17] Douglas JG, Koh W, Austin-Seymour M, Laramore GE. Treatment of salivary gland neoplasms with fast neutron therapy. Arch Otolaryngol Head Neck Surg 2003;129:944-8.

[18] Douglas J, Rainey D, Parvathaneni U, et al. Fast neutron therapy for the treatment of major salivary gland neoplasms. Int $\mathrm{J}$ Radiat Oncol Biol Phys 2012;84:S513-514.

[19] Huber PE, Debus J, Latz D, et al. Radiotherapy for advanced adenoid cystic carcinoma: neutrons, photons or mixed beam? Radiother Oncol 2001;59:161-7. 
[20] Muntner MW, Schulz-Ertner D, Hof H, et al. Inverse planned stereotactic intensity modulated radiotherapy (IMRT) in the treatment of incompletely and completely resected adenoid cystic carcinomas of the head and neck: initial clinical results and toxicity of treatment. Radiat Oncol 2006;1:17.

[21] Pommier P, Liebsch NJ, Deschler DG, et al. Proton beam radiation therapy for skull base adenoid cystic carcinoma. Arch Otolaryngol Head Neck Surg 2006;132:1242-9.

[22] Mizoe J, Hasegawa A, Jingu K, et al. Results of carbon ion therapy for head and neck cancer. Radiother Oncol 2012;103:32-7.

[23] Schultz-Ertner D, Nikoghosyan A, Didinger B, et al. Therapy strategies for locally advanced adenoid cystic carcinomas using modern radiation therapy techniques. Cancer 2005;104:338-44.

[24] Goitein M. Trials and tribulations of charged particle radiotherapy. Radiother Oncol 2010;95:23-31. 